\title{
Accuracy Evaluation of 3D Geometry from Low-Attitude UAV Images: A Case Study at Zijin Mine
}

\author{
Qiuling Wang ${ }^{\text {a }}$, Lixin Wu ${ }^{\text {b,* }}$, Shaojie Chen ${ }^{c}$, Defu Shu ${ }^{\mathrm{d}}$, Zhihua Xu ${ }^{\text {a }}$, Fashuai Li ${ }^{\mathrm{e}}$, Ran Wang ${ }^{\mathrm{e}}$
}

\author{
${ }^{a}$ Key Laboratory of Environmental Change \& Natural Disaster of MOE, Beijing Normal University, Beijing 100875, China \\ ${ }^{\mathrm{b}}$ IOT Perception Mine Research Centre, China University of Mining and Technology, Xuzhou 221008, China \\ ${ }^{\mathrm{c}}$ Resources Department, Longyan College, Longyan 364000, China \\ ${ }^{\mathrm{d}}$ Zijin MiningGroup Co., Ltd, Shanghang 364200. China \\ ${ }^{\mathrm{e}}$ College of Geoscience and Surveying Engineering, China University of Mining and Technology, Beijing 100083, China
}

KEYWORDS: UAV, 3D Geometry, Opencast Mine, Structure from Motion (SfM), Accuracy Evaluation

\begin{abstract}
:
This study investigates the usability of low-attitude unmanned aerial vehicle (UAV) acquiring high resolution images for the geometry reconstruction of opencast mine. Image modelling techniques like Structure from Motion (SfM) and Patch-based Multiview Stereo (PMVS) algorithms are used to generate dense 3D point cloud from UAV collections. Then, precision of 3D point cloud will be first evaluated based on Real-time Kinematic (RTK) ground control points (GCPs) at point level. The experimental result shows that the mean square error of the UAV point cloud is $0.11 \mathrm{~m}$. Digital surface model (DSM) of the study area is generated from UAV point cloud, and compared with that from the Terrestrial Laser Scanner (TLS) data for further comparison at the surface level. Discrepancy map of 3D distances based on DSMs shows that most deviation is less than $\pm 0.4 \mathrm{~m}$ and the relative error of the volume is $1.55 \%$.
\end{abstract}

\section{INTRODUCTION}

Opencast mine is usually in a complex and changeable industrial environment that it is necessary to estimate the exploitation quantity and monitor mine safety. Unmanned aerial vehicles (UAVs) equipped with digital cameras can be used to acquire high temporal and spatial resolution images over traditional remote sensing methods with low-cost and more flexible deployments (Eisenbeiss, et al., 2004). UAV images was utilized successfully to generate ortho-mosaic and digital terrain model (DTM) in landslide investigations and had achieved comparable results as compared to that of terrestrial laser scan data (Niethammer et al., 2011). There are considerable successes in automated reconstruction of 3D point cloud from image sequences in computer vision (CV) (Hartley et al., $2000 \&$ Niethammer el al., 2012). The 3D surfaces and topography accuracy reconstructed from UAV images was evaluated by James et al. (2012) and Douterloigne et al. (2010). It was shown that such low-cost and more flexible approaches can achieve good performance. 3D disaster scene was also reconstructed from UAV images with flight control data (Shen et al., 2011). The scene relative error is less than $0.4 \%$, which demonstrates that the proposed UAV images reconstruction for disaster surveying is feasible and effective. Digital surface models (DSMs) generated from UAV imagery and Terrestrial Laser Scanner (TLS) are compared in a cultural heritage (Eisenbeiss et al. 2006).

In this paper, we recover the scene geometry by implementing the Structure from Motion (SfM) and Patchbased Multi-View Stereo (PMVS) algorithms, generating a couple of point cloud with relative high density. And then, we evaluate the 3D geometrical model at a point level for absolute accuracy assessment. Finally, DSMs generated from UAV point cloud was compared with that from TLS data and the relative error was evaluated.

\section{UAV Platform and Images Acquisition}

\subsection{UAV Platform}

The UAV platform used in this study is a multi-rotor BNU-D81, with eight rotating wings (Fig.1). This platform is stabilized with a consumer digital camera (Canon 5D mark II, 21 Megapixel, 5616×3744 pixels), and the camera's zoom and the aperture are set to be fixed values when aerial navigation. The UAV flight control system is stabilized with inertial measurement units (IMUs) and the endurance is up to 30 minutes using a lithium battery pack with a capacity of $\sim 5 \mathrm{Ah}$. It can be radio-controlled manually or automatically based on the self-learning neural network adaptive control technology (BrainyBEE autopilot system V4.11).

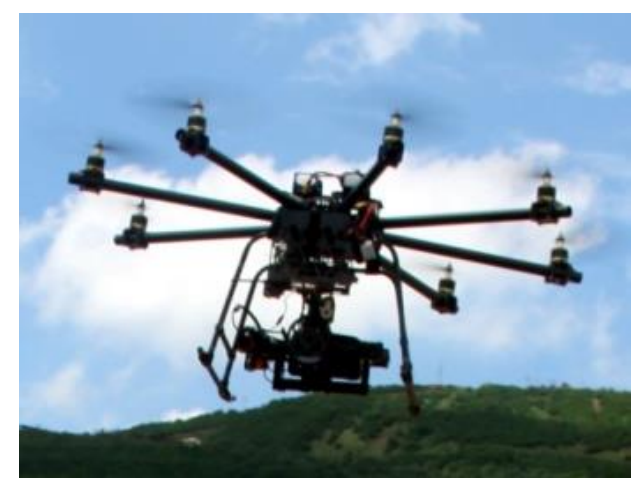

Fig.1. UAV platform BNU-D8-1

\footnotetext{
* Corresponding Author: awulixin@263.net
} 


\subsection{Study area and data acquisition}

The study site is a highly dynamic detritus dump located at Zijin Mine (Fig.2), in Fujian Province, Southeast of China. On July $8^{\text {th }}, 2013$, a UAV flight campaign was carried out at the study area, while a new detritus dump $(100 \times 100 \mathrm{~m})$ with complex topography and large elevation difference was under accumulating. To ensure the whole detritus dump area was covered, the BNU-D8-1 was manually controlled and adjusted its altitude during the flight to keep the distance above the detritus dump remain relatively constant. Manually control of UAV often leads to deviations during the flight navigation, and the deviation of images resolution has to be accepted. The flight is 50 meters above the ground, 32 optical images with high overlaps were acquired. The ground resolution is approximate $0.01 \mathrm{~m}$ per pixel.

17 cross cardboards (the size is $105 \times 297 \mathrm{~mm}$ ) were approximate evenly-distributed over the site and were used as ground control points (GCPs). The cardboards are affixed to the stones and clearly marked in the aerial images. The GCPs were measured by Global Positioning System Real-time Kinematic (GPS-RTK) with an accuracy of $2 \sim 3 \mathrm{~cm}$ in plane and $5 \mathrm{~cm}$ in vertical. However, some of the GCPs were badly appeared in the images and were rejected in the post processing (Eisenbeiss et al. 2005).

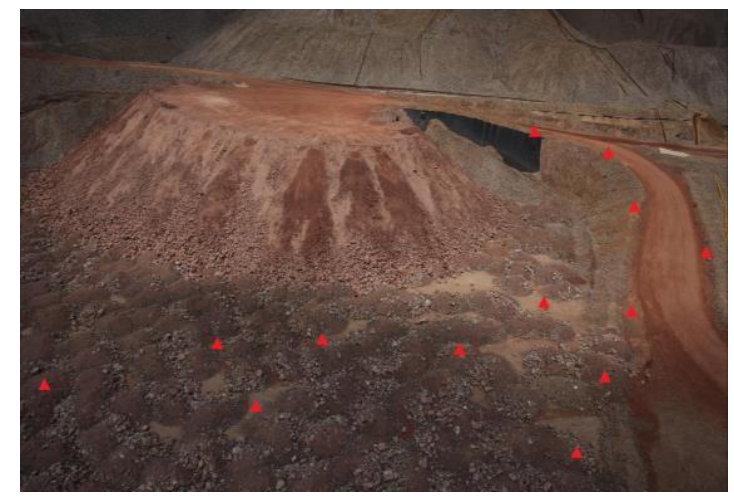

Fig.2 UAV image with GCPs of detritus dump, at Zijin Mine

\section{METHODOLOGY}

\subsection{Processing of UAV images}

In this paper, the UAV images are processed as follows (Fig.3). First, images quality will be checked after UAV images acquired, and remove ghost images or dislocation images in the following process. Then, 3D Geometry of UAV images will be reconstructed using different algorithms. Next, GCPs will be used to register $3 \mathrm{D}$ point could with geographic coordinate system. Finally, the reconstruction model precision will be evaluated with GCPs and TLS data.

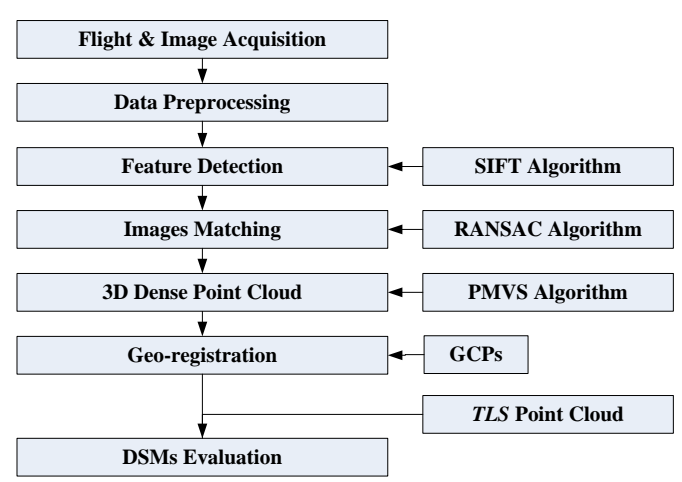

Fig. 3 Workflow for the processing of UAV images

\subsection{D Geometry Reconstruction with UAV Images}

Computer Vision (CV) is highly advanced in recently years and new algorithms have been developed for processing images. Among these algorithms, SfM algorithm is utilized as a cost effective method for processing sets of overlapping UAV images for processing sparse 3D models (Koenderink et al. $1991 \&$ Snavely et al. 2008). The process of SfM refers to three major stages of feature detection, image matching as well as bundler adjustment.

To start the SfM, distinctive features with unique descriptors are first detected with the Scale Invariant Feature Transform (SIFT) algorithm, each of which indexed with a 128 -element vector. And then, Approximate Nearest Neighbours algorithm (ANN) based on K-D tree is used for coarse matching by comparing the Euclidean distance between vectors of potential correspondences.

$F M_{\text {set }}$ is referred to these feature points pairs sets that have matching relations.

$$
\begin{aligned}
& F M_{\text {set }}=\left\{\left(f_{m}, f_{n n}\right)\right\} \\
& f_{n n}=\arg \min \left\|f_{d}(m)-f_{d}(T G)\right\|_{2}
\end{aligned}
$$

Where $f_{m}$ represents target images $P_{m}$ and $f_{n n}$ is the images to be matched $P_{T G}$, respectively.

For each point in the first image of an image pair we search for the best and second best candidates in the other image. In case of the ratio $r=d_{\text {second }} / d_{\text {best }}$ of the certain measures for the second best to the best candidate is lower than a certain threshold. The mismatching possibility is lower when $r$ is smaller. In this paper, we choose $r<0.6$ as potential feasible correspondence.

To further eliminate the mismatching points of coarse matching, Random Sample Consensus (RANSAC) (Fischler et al. 1981) strategy and eight-point algorithms (Hartley et al., 2000) are applied for fine matching. Point cloud will be transformed to plane coordinates with perspective projection. The geometric projection error is used as objective function $g(C, p)$.

$$
g(C, p)=\sum_{i=1}^{n} \sum_{f=1}^{t} w_{i, f}\left\|q_{i, f}-r\left(C_{i}, p_{f}\right)\right\|^{2}
$$




$$
w_{i, f}=\left\{\begin{array}{l}
1, f \in i \\
0, f \notin i
\end{array}\right.
$$

Where

$C=$ camera parameter

$p=$ point cloud coordinates

$n=$ camera points, also refers to the total number of images

UAV acquired

$t=$ fine matching points

The Sparse Bundle Adjustment (SBA) algorithm is applied to solve the nonlinear least squares of objective function $g(C, p)$. The geometric projection error $g(C, p)$ will be reduced by gradually iteration, and optimal camera position as well as flight attitude will be achieved (Snavely et al. 2008). Based on the flight attitude, 3D Geometry of study area can be reconstructed.

In order to improve the level of details in the point cloud, Patch-based Multi-view Stereo (PMVS) algorithm (Furukawa et al. 2009) is further used to obtain the dense point cloud.

\subsection{Geo-registration}

Geo-registration refers to register 3D reconstruction point cloud with geographic coordinate system. The point cloud reconstructed with $\mathrm{SfM}$ is in space rectangular coordinate system. Point cloud can be transformed to WGS-84 coordinate system with measured GCPs. Transformation parameters are computed by Bursa's 7-parameter models and indirect adjustment using three or more common points in both coordinate. Then, 3D point cloud model scan be transformed to WGS-84 space rectangular coordinate.

\subsection{Accuracy Evaluation}

The accuracy of the 3D geometry is evaluated both with GCPs and TLS point cloud. UAV point cloud is first evaluated at the point level by comparing the absolute coordinate between UAV point cloud and GCPs. Further difference is computed with the analysis of DSM difference from both UAV point cloud and TLS. The 3D dense point cloud generated from PMVS allows us to generate high resolution DSMs with RISCAN PRO software.

\section{RESULTS}

\subsection{D point cloud model}

3D dense point cloud is reconstructed with SfM and PMVS algorithm. The density of the point cloud (Fig.4) is 400 points about per square meter.

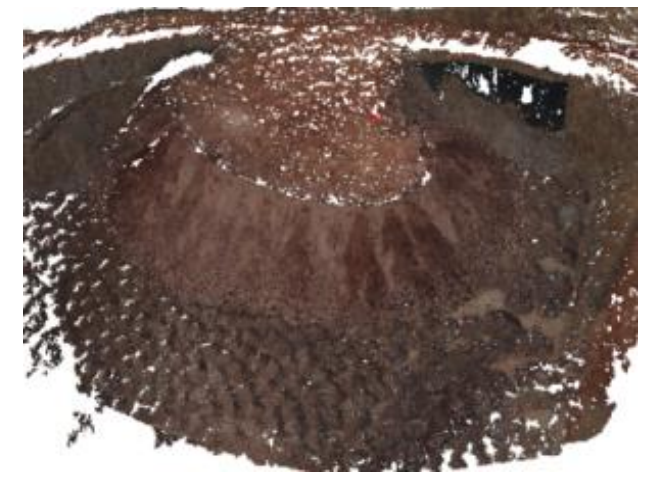

Fig.4 3D dense model of study area

\subsection{Point cloud precision of UAV images}

Comparison the GCPs with UAV points, 12 control points are participated in the experiment. The absolute error of each point was shown in Fig.5. The mean square errors of each direction are $\sigma_{x}=0.07 \mathrm{~m}, \sigma_{y}=0.08 \mathrm{~m}, \sigma_{z}=0.02 \mathrm{~m}$. The compared of GPS measured GPCs and PMVS-based model shows that there is a significant planar error than height direction. The mean square error of point is $\sigma_{p}=0.11 \mathrm{~m}$, which shows that the precision of PVSM-based points can reach to the sub-meter level, which is admitted in mine.

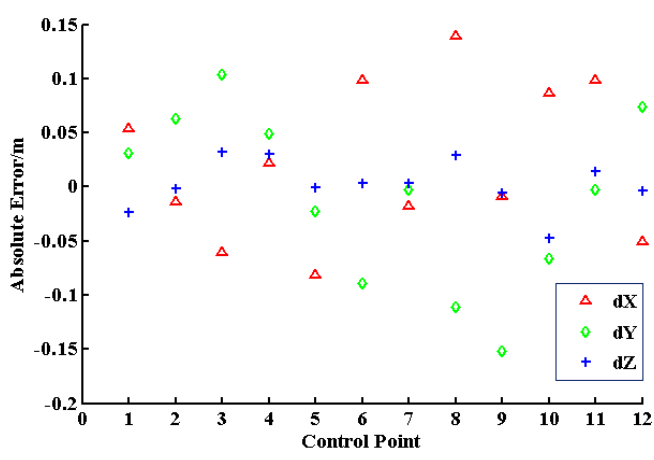

Fig.5 UAV point cloud precision

\subsection{Surface precision evaluation}

In order to evaluate the feature precision of UAV point cloud, Riegl VZ-4000 was used to acquire high resolution point cloud of the detritus dump simultaneously with UAV flight. Fig.6 is the TLS point cloud, where the blind spots and loopholes are due to the limitation of fixed Field of View (FOV).

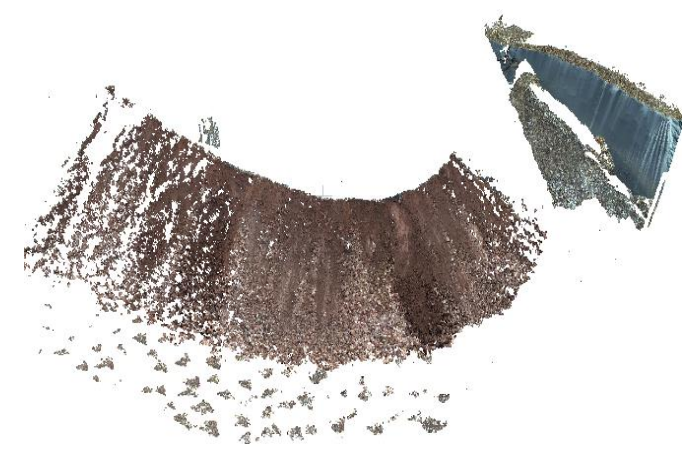

Fig.6 TLS point cloud of study area

Two datasets all include the same side surface areas of detritus dump. These side areas are compared and analyzed with respect to accuracy, resolution and applicability to opencast mine. With manually coarse registration and multi station adjustment using RISCAN PRO software, two surfaces are compared at surface level.

The resolution of UAV point cloud and TLS point cloud are all resampled to $5 \mathrm{~cm}$. Triangulated Irregular Networks (TINs) are first generated based on the two datasets. A discrepancy map of two features was carried out by RIEGL PRO (Fig.7). The 3D comparison of both DSMs shows maximum deviation reaches from $-1.0 \mathrm{~m}$ to $0.98 \mathrm{~m}$, while most deviations are less 
than $\pm 0.4 \mathrm{~m}$. Maximum deviation appeared in local area was due to ore rolling or creeping down during data collection.

Relative precision can be evaluated by the calculating distance between two surfaces. With an $x-y$ auxiliary plane, the volume of these two features can be calculated, and the relative error of volume is $1.55 \%$.

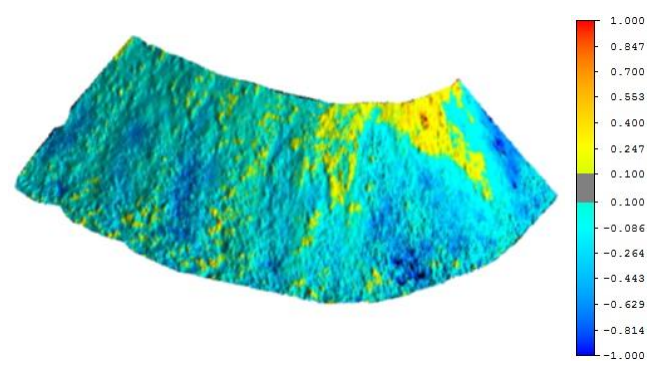

Fig.7 Discrepancy map of 3D distances based on DSMs

\section{CONCLUSIONS}

In this study, low-attitude UAV was applied to acquire high resolution and overlaps images at an opencast mine. With SfM algorithm and PVMS algorithm being applied to images reconstruction, 3D dense point cloud was extracted. RTK and TLS LiDAR were used in the study to evaluate the accuracy of 3D geometry, and geometry precision was evaluated both in point level and surface level. The result shows that both have achieved good performance for mine applications. In future study, UAV will be applied to monitor the opencast mine exploitation quantity.

\section{REFERENCES}

Eisenbeiss, H., 2004. A mini Unmanned Aerial Vehicles (UAVs): system overview and image acquisition. Int. Int. arch. Photogram. Remote Sensing spatial Info, Sci., 36(5/1). (on CDROM).

Niethammer, U., Rothmund, S., Schwaderer, U., Zeman, J., Joswig, M., 2011. Open Source Image-processing Tools for low-cost UAV-based Landslide Investigations. Int. arch. Photogram. Remote Sensing spatial Info, Sci., 38 (1/C22), pp. 161-166.

Hartley, R., Zisserman, A., 2000. Multiple View Geometry in Computer Vision. Cambridge University Press: Cambridge, UK.

Niethammer, U., James, M., Rothmund, S., et al., 2012. UAVBased Remote Sensing of the Super-Sauze Landslide:

Evaluation and Results. Engineering Geology, 128(1), pp. 2-11.

James, M. R., Robson, S., 2012. Straightforward reconstruction of $3 \mathrm{D}$ surfaces and topography with a camera: Accuracy and geoscience application. J. of Geophys. res., 117, F03017.

Douterloigne, K., Gautama, S., Philips, W., 2010. On the accuracy of 3D landscapes from UAV image data. IGARSS, 2010. pp.589-592.
Shen, Y.L., Liu, J., Wu, L.X., et al., 2011. Reconstruction of disaster scene from UAV images and flight-control data. Grography and Geo-Information Science. 27(6), pp.13-17.

Eisenbeiss, H., Zhang, Li., 2006. Comparison of DSMs generated from mini UAV imagery and Terrestrial Laser Scanning in a cultural heritage application. Int. arch. Photogram. Remote Sensing spatial Info, Sci., 36 (5), 90-96.

Eisenbeiss, H., Lambers, K. \& Sauerbier, M., 2005. Photogrammetric recording of the archaeological site of Pinchango Alto (Palpa, Peru) using a mini helicopter (UAV). In Proc. of the 33rd CAA Conference.Tomar, Portugal, 21-24.

Koenderink, J., Vandoorn, A., 1991. Affine structure from motion. Journal of the optical society of America. 8 (2), pp.377385.

Snavely, N. Seitz. S. M., Szeliski, R., 2008. Modeling the World from Internet Photo Collections. International Journal of Computer Vision, 80(2), pp.189-210.

Fischler, M. A., Bolles, R. C., 1981. Random Sample Consensus: A Paradigm for Model Fitting with Application to image Analysis and Automated Cartography. Graphics and Image Processing, 24 (6), pp.381-395.

Snavely N, 2008. Scene Reconstruction and visualization from Internet photo collections. University of Washington.

Furukawa, Y., Ponce, J., 2009. Accurate camera calibration from Multi-View Stereo and Bundle Adjustment. International Journal Computer Vision, 84(3), pp.257-268. 